\title{
Verification Theory of Meaning and the Concept of Time and Space: Implications for Einstein Relativity Theory
}

\author{
Jerome P. Mbat ${ }^{1}$ \& Emmanuel Iniobong Archibong ${ }^{2}$ \\ ${ }^{1}$ Faculty of Arts, Department of Philosophy, University of Uyo, P.M.B 1017, Uyo-Akwa Ibom State, Nigeria \\ ${ }^{2}$ Faculty of Arts, Department of Philosophy, University of Uyo, P.M.B 1017, Uyo- Akwa Ibom State, Nigeria \\ Correspondence: Emmanuel Iniobong Archibong, Faculty of Arts, Department of Philosophy, University of Uyo, \\ P.M.B 1017, Uyo- Akwa Ibom State, Nigeria. E-mail: emmymark2jr@yahoo.com
}

Received: December 5, 2012

Accepted: January 23, 2013

Online Published: March 11, 2013

doi:10.5430/elr.v2n1p14

URL: http://dx.doi.org/10.5430/elr.v2n1p14

\begin{abstract}
The verifiability theory of meaning was introduced to delineate scientific statements from metaphysical ones via language. The project was intended to make for clarity of meaning of words especially as it is held that most statements we make are useless, meaningless and out of context. This work attempts to show, by way of analysis, that applying this theory to the concept of time and space in Einstein theory of relativity, reveals that even though the verifiability criterion for truth and meaning is founded upon observational statements, it creates more confusion than proffer solution to our general understanding of time and space by reducing it to a subjective private language affair relative to an observer.
\end{abstract}

Keywords: Verifiability, Meaning, Relativity theory, Time and space, Truth

\section{Introduction}

The importance of meaning in the use of words and language cannot be quantified. Without making clear the meaning of the words we use, communication will be vague. Meaning brings about clarity and gives light to the understanding. This of course explains why we have a lot of theories as regards meaning. Some of these theories fall under the category of denotation or connotation.

The Logical Positivists, otherwise known as the "Vienna circle" are a group of philosophers, mathematicians and scientists, who gathered in Vienna in the 1920's. These men felt that philosophy in the past had been largely given over to useless controversy over metaphysical and normative problems that were in principle insoluble (including such problems as those of time and space).

The quest of this group was a very laudable one because of the importance of making sense when we communicate using language, especially for the purpose of clarity. But we shall discover that despite their noble quest, the solution they proffer in terms of the principle of verifiability as a criterion for truth and meaning, multiplied even more, the problem of meaning when it is analyzed linguistically in relation to Einstein's theory of relativity and his interpretation of time and space. Let us begin our discourse by examining briefly the "theory of meaning".

\section{Theory of Meaning}

The traditional doctrine of "meaning" possesses extension (the sets of things a term is true of) and intension (something internal or mental concepts). Thus traditional semantic theory leaves out only two contributions to the determination of extension- the contribution of society and the contribution of the real world.

Sifting out linguistic meaning from words requires philosophical analysis. What are we saying about a linguistic expression when we specify its meaning? This is the basis of analysis. The three modes of explanation of meaning are the referential, the ideational and behavioural. The question to be posed here is: do all meaningful expressions refer to something? Alston (1964) opines that:

If the referential theory of meaning is based on the fundamental insight that language is used to talk about things, the ideational and behavioural theories are based on an equally fundamental insight that words have the meaning they do only because of what human beings do when they use language (p.19). 
Every meaningful linguistic expression as exemplify in the referential theory of meaning encounters problems even in those areas where the referential theorist feels more secure. This, of course, only goes to show that alternative mode of meaningful explanation of words via language has to be devised. The verifiability theory of meaning is a referential theory by its presuppositions and assumptions. It is also seen as incorporating the canons of empirical science. These will be made clear shortly and that will be our major focus in this work as far as theory of meaning is concern.

\section{The Principle of Verification as a Theory of Meaning}

The principle of verifiability arose as an attempt to solve the problems of the demarcation of science. That is, to resolve the question about when a theory should be ranked as scientific or non- scientific; the problem of whether there is a criterion for the scientific character or status of a theory. The problem was reduced by Popper to the problem of drawing a distinction between science and pseudo-science (metaphysics) that is, what is "science" or "scientific" and non-scientific or pseudo-science "knowing very well that science often errs, and that pseudo-science may happen to stumble on the truth" (Popper, 1963:p.33).

Traditionally science is distinguished from pseudo-science or metaphysics by its empirical method which is essentially inductive, proceeding from observation or experiment. It was Popper who, on the contrary formulated the problem as one of demarcation between a genuinely empirical method and a non-empirical one or even a pseudo-empirical method. That is, a method which, although appeals to observation and experiment, nevertheless does not come up to scientific standard, especially in terms of their explanatory power. Thus by pseudo-empirical method we shall refer to it as one that though posing as scientific, has in fact more in common with primitive myths than with science, one that resemble astrology rather than astronomy (Popper, 1943:p.34).

Thus from the inductivist point of view, characteristically, it is the incessant streams of confirmation, of observations which verifies theories. That is to say the truth of any scientific theory rests ultimately on its being constantly verified or verifiable by observations. And there are always multi-instantiations of the operation of any theory in nature. This of course, constitutes the strongest argument in favour of the traditional meaning/understanding of scientific truth and theory and even of statements.

This concern was to purge philosophy of all metaphysics since metaphysical disputes are devoid of empirical significance. Hence the major proposal of the members of the Vienna Circle was that empirically significant statements are verifiable statement if and only if it is possible to specify conditions which would make the statement true; and it was believed that metaphysical statements could not satisfy this requirement.

Moreover, to meet the dispute that soon arose over the meaning of the phrase "possibility of verification", there was a general agreement that a statement is empirically significant only if it is logically possible to verify it. But another sense of "possibility of verification" was developed by Reichenbach (1953) when he spoke of the "physical possibility" of verification (p.97).

In his view, it is physically possible to verify a statement only if a verification procedure can be specified which does not violate accepted scientific laws. His suggestion was that the verifiability criterion be interpreted so that empirical significance is given only to statements for which verification is both logically and physically possible.

An application of this criterion, of course, would exclude for instance, speculation about the spectrum of a nebula receding from the Earth with a velocity greater than that of light. It is not logically impossible that information be transmitted with a velocity greater than that of light, but we believe it to be physically impossible. And, of course, the scientists' idea about physically possible processes does change and we suppose that Reichenbach did recognize that application of verifiability criterion is relative to the state of development of science at any time.

However, the verifiability criterion soon was not recognized to be overly restrictive because it excludes universal statements such as "all sodium samples react with chorine" and "all negatively charged bodies attract positively charged bodies". No finite set of observation reports could establish these statements as true. The most that can be established is that no exceptions have been observed to date. But since many important scientific laws are universal statements of this type, the proposed criterion of demarcation is hardly adequate.

The situation, however, will strikingly be different from theories studied in the natural sciences as exemplified by Newton's theory and especially from Einstein's theory of relativity. Consider Popper's (1963) example of Einstein's gravitational theory which led to the prediction that light must be attracted by heavy bodies, such as the sun, just as material bodies were attracted. From this prediction it became possible to show by calculation that the light from distant fixed star whose apparent position was close to the sun would reach the earth from such a direction that the star would seem to be slightly shifted away from the sun. That is to say, that stars close to the sun would appear as if they had 
moved a little away from the sun and from one another. This prediction could be claimed to be exact as it is based on mathematical calculations.

But Popperian and other positivists' problem is the risk involved in a prediction based on observation on the account of the possibility of the predicted effect of observation being different or even absent. Thus:

If observation shows that predicted effect is definitely absent, then the theory is simply refuted. The theory is incompatible with certain possible results of observation (Popper, 1963: p.36).

Informed by this risk, Popper came to the conclusion as summarized below that:

(i) It is easy to obtain confirmations, or verifications, for nearly every theory if we look for confirmations he remarked.

(ii) Confirmations should count only if they are the result of risky predictions; that is to say, if unenlightened by the theory in question, we should even expect an event which was incompatible with the theory-an event which would have refuted the theory.

(iii) Every "good" scientific theory is a prohibition; it prohibits certain things to happen. The more a theory prohibits, the better it is.

(iv) A theory which is not refutable by any conceivable event is non-scientific. Irrefutability is not a virtue of a theory (as people often think) but a vice.

(v) Every genuine test of a theory is an attempt to falsify it. Testability, therefore, is synonymous to falsifiability although there are degrees of testability, in the sense that some theories are more testable and exposed to refutation or risks than others.

(vi) Thus supporting evidence should count only when it is the result of a genuine test of a theory; in other words when "it can be presented as a serious but unsuccessful attempt to falsify the theory.

Some genuinely testable theories, when found to be false are still upheld by their admirers by resorting to a conventionalist stratagem, through introducing "ad hoc" auxiliary assumption, or by re-interpreting the theory "ad hoc" in such a way that it escapes refutation. In sum, the criterion of the scientific status of a theory is its falsifiability, refutability, or testability according to which statements or systems of statements in order to be ranked as scientific, must be capable of conflicting with possible or conceivable observations.

\section{Falsifiability as the Criterion of Scientific Status of a Theory}

Wittgenstein (1974) sees metaphysical or philosophical propositions as non-propositions or pseudo-propositions in the sense that they are "senseless" or meaningless. He opined thus:

All genuine (or cognitively meaningful) propositions were truth functions of the elementary form of atomic propositions which described "atomic facts" - that is, facts which can in principle be ascertained by observation (p.54-55).

In other words, meaningful propositions are fully reducible to elementary or atomic propositions which are simple statements describing possible states of affairs, and which could in principle be established or rejected by observation.

In the same vein, Wittgenstein argues that we call a statement an "observation statement" not only if it states an actual observation but also if it states anything that may be observed. We shall have to equally say that every genuine proposition must be a truth-function of, and therefore deducible from observation statements. All other apparent propositions will be meaningless or pseudo-propositions; in fact they will be nothing but nonsensical gibberish (Popper, 1963: p.39-40).

\subsection{Special Theory of Relativity}

This theory also known as STR is the physical theory of measurement in inertial frames of reference. It generalizes Galileo's principle of relativity that all uniform motion is relative and that there is no absolute and well-defined state of rest (no privileged reference frames) from mechanics to all the laws of physics, including both the laws of mechanics and the laws of electrodynamics, whatever they may be.

Pagels (1982) avers that it is "the interplay between the relativity of motion for all material objects and the absoluteness of the speed of light which is at the root of all the unfamiliar features of the world according to special relativity" (p.18). Put differently, special relativity incorporates the principle that the speed of light is the same for all 
inertial observers regardless of the state of motion of the source. The special theory of relativity is the result of two postulates:

1) The laws of physics are the same in all inertial reference frames. The laws of mechanics are the same for all observers in uniform motion.

2) The speed of light in empty space is the same for all inertial frames. The speed of an object depends on the reference frame; however the speed of light in space is the same.

Why does the universe appear to have one time and three space dimensions? In other words, what are space and time, and what is the strange combination of space and time that is called the Space-time Continuum? Space-time continues to be one of the great scientific mysteries of the universe. For Hawking (1988) "we must accept that time is not completely separate from and independent of space, but is combined with it to form an object called space-time" (p.26).

There is another underlying question we have never answered, it is best illustrated thus: if we travel to the end of the universe and find a brick wall, what is behind the wall? Is our universe inside a walled "container" that might allow us to measure distances from points on the fixed surface of the container? Or is our universe the container itself with nothing beyond the boundaries? If so there is no fixed background we can use to measure time and space. General relativity favors a background-free universe where distance and time are measured by relative positions of objects in our universe so that in a real sense what time it is depends on which object we are standing on when we look at our watch. Quantum Theory favors (but does not necessarily require) the existence of a complex, yet still fundamental time.

What then is the fundamental nature and origin of all the numbers that describe our universe, like the value of "pi" in the radius of a circle, including space-time? It is clear that human beings cannot go beyond what science calls boundary conditions, yet it may also be impossible for us to answer questions about the most fundamental physical nature of our observable universe.

The special theory of relativity has a wide range of consequences which have been experimentally verified including counter intuitive ones such as length contraction, time dilation and relativity of simultaneity which contradict the classical notion that the duration of the time interval between two events is equal for all observers. On the other hand, it introduces the space-time interval which is invariant.

Combined with other laws of physics, the two postulates of special relativity predict the equivalence of matter and energy as expressed in the mass energy equivalence formular $\mathrm{E}=\mathrm{Mc}^{2}$ where $\mathrm{C}$ is the speed of light in a vacuum. The theory was originally termed "special" because it applied the principle of relativity only to the special case of inertial reference frames; that is, frames of reference in uniform relative motion with respect to each other.

\subsection{General Theory of Relativity}

This theory, also known as GTR, treats non-inertial systems, that is, those system accelerating relative to each other (Blackburn, 2004: p.315). The leading idea is that the laws of motion in an accelerating frame are equivalent to those in a gravitational field (Christian, 2009:p.512). The theory treats gravity not as a Newtonian force acting in an unknown way across distance, but as a metrical property of a space-time continuum that is curved in the vicinity of matter. Because acceleration bends light and because acceleration and gravitation are equivalent, Einstein reasoned that gravitation should bend light too.

And there the idea rested, without support from observation or experiment, until 1919, when two English expeditions were dispatched to Brazil and the African Island of Principe to observe a solar eclipse. The hope was to find out if starlight would be bent as it passed close by the sun's massive body; only during an eclipse could light from distant stars be seen. Measurements confirmed Einstein's calculations as the predictions turned out to be so (Christian, 2009: $\mathrm{p}$. 514).

From the equivalence principle, Einstein made important inferences. Among them were that clocks would be seen to run at different rates in different gravitational fields, and that light radiating from a massive body, like the sun or a star, would lose some of its energy. Confirmation of both inferences would have to wait for the development of more sophisticated technologies. Other related inference were that, the universe is expanding, and that the far part of it is moving away from us, faster than the speed of light and a rotating mass 'drag along' the space time around them, a phenomenon termed "frame-dragging". 


\section{The Philosophical/Linguistic Problems of the Concept of Time and Space}

The concept of time and space generates serious philosophical problems upon reflection and a linguistic one, too, because of its confused nature and perhaps due to the ambiguity of the words "time and space". Christian (2009) observes that: "Time is intimately familiar and ultimately formidable" (p.235).

An attempt to understand the content of the concept of time and space betrays the frailty of our thoughts and the ineptness of our language. Often the confusion that surrounds the concept of time is its application to a variety of functions. We communicate with one another using the word time and space in pragmatic sense without really giving it a thought, what we are talking about. Christian (2009) again makes the point that philosophically speaking, the following questions about time are:

What is time? How do we experience it? Can we understand it? What is meant exactly by "past", "present", and "future"? In what sense can each of them be said to exist? Where in time do we live? What does time have to do with personal existence? (p. 236).

An attempt to sincerely answer the following questions throws us into a deep confusion that reveals the limit of human cognition. This is why Gale (1968) calls the problem of time "the most important and exciting issue in all of philosophy" (p. vii).

The first serious attempt to analyze the concept of time occurs in Aristotle's Physics. He raises the question "in what sense can time be said to exist"? Relating the notion or idea of existence to time creates a dilemma. In the first instance, we have an idea of time, whether it has to do with the workings of the rotation of the universe that leads to day and night, or the clock-work or (chronological time). On the other hand, only individual substances which are compounds of form and matter can be said to exist in an unqualified sense; everything else are attributes of these substance. Is time then an attribute or a substance?

Some thinkers like St. Augustine sees time to be a "protraction" of the mind and real existence should not be conferred to it because it cannot be extensively defined. Thus Gale (1968) opines that "the question what is time is not a question about natural science" (p. 5). There are many surface grammatical analogies between temporal expressions and expressions that refer to physical objects and processes. Hence, when we try to use temporal expressions to refer to time, we would be trying to "catch the shadows cast by the opacities of speech" (Waismann, 1956: p.2).

When we use the word "time" at least it relates to three different phenomena, all quite distinct. The first is the clock time or chronological time (a derivative of Greek chronos meaning "time"). But here, clocks only measure space; that is, the movement of the sun from one point to another. The clock on the wall is set to correlate with the sun's motion, but we are not synchronizing time.

The second use of the phenomenon of time refers to a kind of subjective time (Psychological or experiential time). Psychological time is our individual experience of the continuum of our consciousness. Thus consciousness plays an important role in the understanding of time. When we are asleep or unconscious, time becomes non-existent. But time begins in the moment we regain consciousness.

A third kind of phenomenon that we think of as "time" is matter-in-motion that is, sequence of events occurring in the real world. Here the sun rises, rain falls, and waves break upon the shores and so on. It is only natural to use the word time to refer to such real events while we measure such event against our calibrated clocks or experiential time. It is glaring from our analysis that the concept of time does generate some serious philosophical confusion in language. The problem is akin to what Udo Etuk (2001) envisaged when he penned down the following words:

Can we remember the future? That is to say, can there be anything in our experience which is analogous to memory but with respect to the future - a kind of remembering forward? ( $\mathrm{p}$. 1).

Space just like time is without its antecedent problems in language. It is a concept that prima facie, appears simple but on a serious philosophical reflection, we might find out that space may as well be an illusion. Various thinkers have theorized about the concept of space. However, Isaac Newton distinguished between absolute space and relative space. This is how he shows the distinction in Gribanov (1987):

Absolute space in its own nature, without relation to anything external, remains always similar and immovable. Relative space is some movable dimension or measure of the absolute spaces which our senses determine by its position to bodies, which are commonly taken for immovable space (p. 6). 
From the afore-quoted, space is independent of matter. Space then is seen as a vacuum that matter fills up, even though it is different in property and in its existence. This is the point at which all the theorizing about space meets. But the question still remains: is space independent of matter or does matter gives us an idea of space? Put differently, can there be space without matter or matter without space? It is from this notion that space is separated from time and vice versa.

\section{Evaluation and Conclusion}

The members of the "Vienna circle" or the positivists who came up with the "verifiability theory of meaning" opines that in order for one to be making sense, one must be able to specify the way in which what he says can be empirically verified. Thus to them, observation plays a very crucial role in the determination of truth, hence every sentence in question must be capable of an empirical test. If we apply this criterion in strict sense we would discover that the whole idea of space-time in Einstein's relativity theory will be fogged with ambiguity especially where space and time become relative to the observer. Einstein once defines time as what we measure with a clock and space as what we measure with a rod (Pagels, 1982: p.16). For Hawkins (1988) each person has his own personal measure of time depending on where he is and how he is moving (p. 36). If this is an intelligible position, then the Scientists' claim of objectivity remains suspect.

Determining truth about Space-time in Einstein relativity theory might pose a hard nut to crack for the philosopher and the linguist. At best what we may get is relative idea of time and space and there may be no general concession. Truth about reality especially of space and time will then be relative and to these "... anything goes" (Fereyabend, 1975: p.296). Also since the universe is not static and time flows, in trying to catch up with time in order to understand it will mean to wish for time to come to a halt. As Popper (1963) puts it:

I thus felt that if a theory is found to be non-scientific, or

metaphysical (as we might say) it is not thereby found

to be unimportant or insignificant, or meaningless or

nonsensical (p.38).

In conclusion, this work has been an attempt to show the pitfalls in the verificationist project of meaning especially as they tried to separate science from metaphysics. Their criterion for truth was verification, confirmation and observation. But if we subject these criteria to linguistic scrutiny, we will discover, like we have done that we may reach a dead end because such concepts as time and space or space-time (following Einstein) are highly metaphysical and abstract in nature. Trying to describe them using observational statements, multiplies its meaning especially since Einstein holds that time and space are relative to an observer and the quest to unravel it will remain shrouded in mystery that bewilders the human mind.

Moreover, from our detailed analysis of verifiability as a criterion of empirical truth, it is clear that the criterion is overly restrictive as it excludes universal statements including statements of Einstein relativity theory. Hence no finite set of observation reports could establish its truth.

Similarly, from Charles Sanders Pierce conception of truth, Einstein has no guarantee that any particular belief including belief in his relativity theory will ever be immune from the need to be revised; this point to the fallibility of all statements including the ones expressing Einstein's relativity theory. The logic is that “ $\ldots$ if we think we have arrived at an infallible truth, this would put an end to inquiry, which is essential to the self-correcting nature of the scientific method" (Lawhead, 2002: p.466).

But granting the plausibility of fallibilism that absolute truth does not exist except as the ideal goal of inquiry, we can, at least hope for the truth that continue to work for the time being until experience causes us to revise them.

\section{References}

Alston, W.P. (1964). Philosophy of language. New Jersey: Prentice-Hall.

Ayer, A. J. (1963). Language, truth and logic. Middlesex: Penguin Books.

Blackburn, S. (2005). Oxford dictionary of philosophy. Oxford: Oxford University Press.

Christian, J. L. (2009). Philosophy: an introduction to the art of wondering (10 ${ }^{\text {th }}$ ed.) Belmont: Wadsworth Centage.

Feyerabend, P. (1975). Against method: outline of an anarchistic theory of knowledge. London: New Left Books.

Gale, R.M (ed). (1968). "What is time" in: The philosophy of time. London: Macmillan.

Gribanov, D.P. (1987). Albert Einstein's philosophical views and the theory of relativity. (Trans.) H. Campbell. Moscow: Progress Publishers. 
Hawking, S. (1988). A brief history of time. London: Bantam Press.

Lawhead, W.I. (2002). Voyage of discovery: a historical introduction to philosophy. $2^{\text {nd }}$ ed. Belmont: Wadsworth-Thomson Learning.

Pagels, H.R. (1983). The cosmic code: quantum physics as the language of nature. New York: Bantam Books.

Popper, K. R. (1963). Conjectures and refutations: the growth of scientific knowledge. London: Routledge and Kegan Paul.

Reichenbach, H. (1953). "The verifiability theory of meaning" in: Readings in the philosophy of science (ed) H. Feigl \& M. Broadbeck. Newyork: Appleton-Century-Croft.

Udo E. A. (2001) "Remembering the future” in: Sophia: an african journal of philosophy. 3(2) pp.1-10.

Waismann, F. (1956). "How i see philosophy" in: Contemporary british philosophy (3 ${ }^{\text {rd }}$ ed) H.D. Lewis (ed.) London: Blackwell Publishers.

Wittgenstein, L. (1974). Tractatus logico-philosophicus (Trans.) D. F. Pears \& B. F. McGuiness. London: Routledge. 